\title{
SCAR marker for sex identification of Pistacia chinensis Bunge (Anacardiaceae)
}

\author{
Q. Sun, X. Yang and R. Li \\ Department of Biology, Qingdao University, Qingdao, China \\ Corresponding author: R. Li \\ E-mail: 1rg@qdu.edu.cn
}

Genet. Mol. Res. 13 (1): 1395-1401 (2014)

Received January 17, 2013

Accepted July 27, 2013

Published February 28, 2014

DOI http://dx.doi.org/10.4238/2014.February.28.12

\begin{abstract}
Pistacia chinensis Bunge is a dioecious plant that originated in China, and its sex cannot be identified at the early stage of cultivation by only its appearance. Recent studies show that the seed of $P$. chinensis is an ideal feedstock for biofuel production. To guide the cultivation of this energy plant scientifically, a new method is urgently needed to identify the sex of $P$. chinensis seedlings. In this paper, from 21 random-amplified polymorphic DNA primers and 20 inter-simple sequence repeat primers, 2 sex-specific primers (S1 and S281) were identified that can amplify female-specific fragments of 473 and 1242 bp, respectively. However, only 1 fragment (FS281) was converted successfully into a sequence-characterized amplified region marker using S281-1 and S281-2 primers. When the annealing temperature was $64^{\circ} \mathrm{C}$, a 636-bp specific sequence appeared in all female specimens but was absent in all the male samples tested. This study will offer some clues to sex selection in P. chinensis plantations.
\end{abstract}

Key words: Sex determination; Random-amplified polymorphic DNA; Inter-simple sequence repeat; Sequence-characterized amplified region; Pistacia chinensis 


\section{INTRODUCTION}

Many flowering plant species in nature are hermaphrodites, which have both stamens and pistils in the same individual. However, there are also many dioecious plant species, in which female and male sex organs grow on different individuals. It is estimated that dioecious plants account for $6 \%$ of all flowering plants (Renner and Ricklefs, 1995). Pistacia chinensis, a dioecious plant species, is a member of the Anacardiaceae family. There are several distinctive features of $P$. chinensis, e.g., it can grow in environments of dry, acidic, or alkaline soil conditions, and it is widely distributed in China (Qin et al., 2010). In addition, the oil content of the P. chinensis seed is generally above $30 \%$, and up to $82 \%$ of the oil is unsaturated fatty acid (Li et al., 2012). Recently, there has been a growing interest in developing biofuels because of the shortage of fossil oil. Based on the advantages of its wide distribution and high yield of oil-rich seeds, $P$. chinensis has been an important raw material for biodiesel production in many parts of China.

To obtain the maximum economic benefits from large-scale plantations of $P$. chinensis, the proportion of male and female plants should be controlled in a reasonable scope when they are planted at the very beginning. However, until now, there is no one reliable method to identify the sex of the individual plants of $P$. chinensis, especially at the seedling stage. Therefore, it is urgent to develop an effective method to identify the gender of $P$. chinensis at the seedling stage. In recent years, molecular biological methods have been widely used in plant sex identification, in which random-amplified polymorphic DNA (RAPD)-based technology has played an important role in identifying the sex of the dioecious plants and animals (Maki, 2009; Heideman et al., 2010), and sequence-characterized amplified region (SCAR) markers, improved from RAPD markers, were proven to be more effective. At present, a female-linked SCAR marker has been successfully used to discriminate the sexuality of $P$. vera L., which belongs to the same genus as $P$. chinensis (Yakubov et al., 2005). In this paper, we aimed to develop a reliable and reproducible marker for the sex identification of $P$. chinensis to lay the foundation for reasonable planting of this oil plant in a large scale.

\section{MATERIAL AND METHODS}

\section{Plant and genomic DNA extraction}

Leaf samples of $P$. chinensis were collected from 8 trees including 4 male trees and 4 female trees that were grown in Zhongshan Park and 8 trees including 4 male trees and 4 female trees that were grown on Laoshan Mountain in Qingdao in April 2012. Plant sex was determined according to characteristics of its inflorescences. Genomic DNA was extracted according to the protocol that was supplied with the Plant Genomic DNA kit (Tiangen, Beijing, China).

\section{RAPD-polymerase chain reaction (PCR) and inter-simple sequence repeat (ISSR)-PCR}

A total of 41 primers, including 21 decamer random primers (Table 1) and 20 ISSR primers (Table 2), were used to screen the sex-specific DNA fragments by PCR. A $25-\mu \mathrm{L}$ PCR 
mixture contained the following: $1 \mathrm{U}$ Taq DNA polymerase, $25 \mathrm{ng}$ genomic DNA, $2.5 \mu \mathrm{L} 10 \mathrm{X}$ buffer (200 mM Tris-HCl, pH 8.4, $200 \mathrm{mM} \mathrm{KCl,} 100 \mathrm{mM}\left(\mathrm{NH}_{4}\right)_{2} \mathrm{SO}_{4}$, and $\left.15 \mathrm{mM} \mathrm{MgCl} 2\right), 0.8$ $\mathrm{mM}$ each dNTP, and $0.8 \mu \mathrm{M}$ RAPD or ISSR primers. RAPD-PCR and ISSR-PCR were carried out under the following conditions: $94^{\circ} \mathrm{C}$ pre-denaturation for 4 min followed by 35 cycles of $94^{\circ} \mathrm{C}$ for $30 \mathrm{~s}, 40^{\circ} \mathrm{C}$ for $45 \mathrm{~s}$, and $72^{\circ} \mathrm{C}$ for $10 \mathrm{~min}$.

\begin{tabular}{llll}
\multicolumn{2}{l}{ Table 1. Primers and nucleotide sequences used in RAPD. } & & \\
\hline Primer & Sequence & Primer & Sequence \\
\hline S1 & GTTTCGCTCC & S12 & CCTTGACGCA \\
S2 & TGATCCCTGG & S279 & CAAAGCGCTC \\
S3 & S281 & GTGGCATCTC \\
S4 & SATCCCCCTG & S282 & CATCGCCGCA \\
S5 & GGACTGGAGT & OPA04 & GGCTGCAATG \\
S6 & TGCGCCCTTC & OPA10 & AATCGGGCTG \\
S7 & TGCTCTGCCC & OPO08 & GTGATCGCAG \\
S8 & GGTGACGCAG & OPT06 & CCTCCAGTGT \\
S9 & GTCCACACGG & OPN11 & CAAGGGCAGA \\
S10 & TGGGGGACTC & & TCGCCGCAAA \\
S11 & CTGCTGGGAC & & \\
\hline
\end{tabular}

Table 2. Primers and nucleotide sequences used in ISSR.

\begin{tabular}{|c|c|c|c|}
\hline Primer & Sequence & Primer & Sequence \\
\hline UBC801 & $(\mathrm{AT})_{8} \mathrm{~T}$ & UBC 811 & $(\mathrm{GA})_{8} \mathrm{C}$ \\
\hline UBC 802 & $(\mathrm{AT})_{8} \mathrm{G}$ & UBC 812 & $(\mathrm{GA})_{8}^{8} \mathrm{~A}$ \\
\hline UBC803 & (AT) $\mathrm{C}$ & UBC 813 & (CT) $\mathrm{T}$ \\
\hline UBC 804 & $(\mathrm{TA})_{8} \mathrm{~A}$ & UBC 814 & (CT) ${ }_{8}^{8} \mathrm{~A}$ \\
\hline UBC 805 & $(\mathrm{TA})_{8} \mathrm{C}$ & UBC815 & $\left(\mathrm{CT}_{8}^{8} \mathrm{G}\right.$ \\
\hline UBC806 & $(\mathrm{TA})_{8} \mathrm{G}$ & UBC816 & $(\mathrm{CA})_{8}^{8} \mathrm{~T}$ \\
\hline UBC807 & $(\mathrm{AG})_{8} \mathrm{~T}$ & UBC 817 & $(\mathrm{CA})_{\mathrm{B}} \mathrm{A}$ \\
\hline UBC808 & $(\mathrm{AG}) \mathrm{C}$ & UBC 818 & $(\mathrm{CA}) \mathrm{G}$ \\
\hline UBC809 & $(\mathrm{AG})_{8} \mathrm{G}$ & UBC819 & (GT) $\mathrm{A}$ \\
\hline UBC 810 & $(\mathrm{GA})_{\mathrm{T}}^{\mathrm{T}}$ & UBC 820 & (GT) $\mathrm{C}$ \\
\hline
\end{tabular}

The female-specific DNA fragments were extracted and purified using the Agarose Gel Extraction Kit and PCR Clean-up Kit (Sing Fo International, Hong Kong, China). The extracted DNA was ligated into the pMD18-T vector (Takara, Dalian, China) and sequenced.

\section{PCR amplification of female-specific SCAR markers}

Two pairs of primers were designed by the DNAman software according to the isolated DNA sequences. The pair of primers that was used for FS1 was S1-1 (5'-CGCTCCTTCTAAT GTTGATGACAA-3') and S1-2 (5'-TCGCTCCCTCCAAATCCAATAAAC-3'). The other primers that were used for FS281 were S281-1 (5'-CCTGGTTGCTTGTGTTGATTAG-3') and S281-2 (5'-GAGTGTCATCAAGCCATCTGTC-3'). The SCAR-PCR system was the same as that described above except that the forward and reverse primer concentrations were both 0.4 $\mu \mathrm{M}$. The program was the same as that described above except that the annealing temperature was $64^{\circ} \mathrm{C}$. 


\section{RESULTS AND DISCUSSION}

\section{RAPD and ISSR analysis}

Of the 41 primers, only 2 RAPD primers, S1 and S281, could amplify sex-specific fragments of 473 and $1242 \mathrm{bp}$, which were named FS1 and FS281, respectively (Figure 1). Both primers were used to test 16 different individuals including 8 male trees and 8 female trees, and FS1 and FS281 could be amplified in all of the female specimens (Figure 2). After sequencing, the sequences of FS1 and FS281 were deposited in GenBank: KC149529 and KC137928. Of the 20 ISSR primers, 6 primers (UBC801, UBC802, UBC803, UBC804, UBC805, and UBC806) could not amplify any band using either male or female individual genomic DNA, and the others could not amplify any sex-specific fragment.

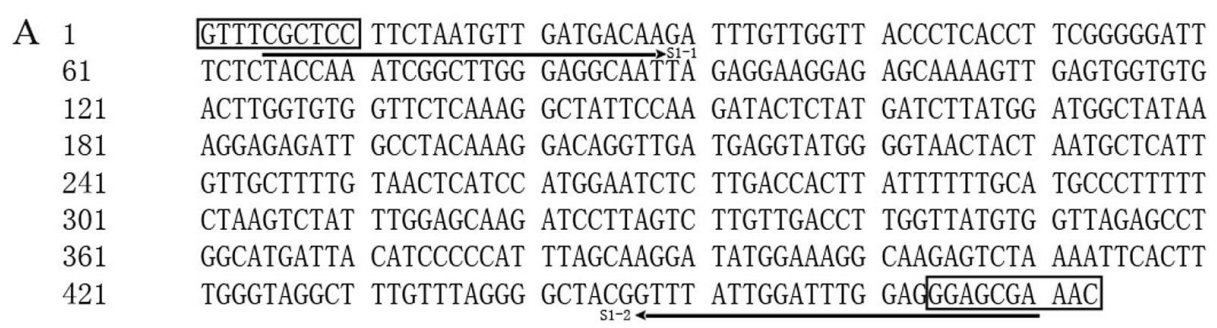

B 1 GTGGCATCTC AACGAGGGCG ACTGAGTTGA CGAATGGAGA GTTGGCATAG CTCTGAGGCA 61 GGCTCTTCTT GATTGGAGTT AAGACTCTAG GAACTCGTTG GATCATCGCC TTTATCTCTA 121 CTAGCCTCTT AGCCATTGTT TATTTTAGGG CGATTTTGGC TGACAAAAAA AGGTGTACAC 181 AAGTGGATGC AAAAGTTGTG GGATTGTCGC TCGATGTGAG TAGTATAGTT GACCTGGTTG 241 CTTGTGTTGA TTAGTGAATT AGAATGGTCA ACTTGAATTG CATAATTAGA GTCGGCTAGA 301 TGAATGAAGG CAAAGTCGAC TGGATGAATG GGGGCAGAGT TGAGCAAAGT CGACTGGATG 361 AGCCGAGGTG GAGCCGATTG GATGAGTGGA GGCAAAGCTT ACTAGATGAG TAAAGGCAGA 421 GCAGAGTCGA TTGGATGAGT ATAGGTGAAG CTGACTGGAT GAGTGAAGGC AGAGCCAACT 481 AGATGAGTAG AGGAGGAGCC AATTGGATGA GTGAAGGCAA AGCCGACTAG ATGAATGGAG 541 GCAGAGTCGA GCAAAGTTGA GCGGAGTCAA TTATACGAGT AGAGATAGAG CCGACTGGAC 601 AAGTGGAGAC GGAGTCCACT GGAAAAGAGA AGACATAACT AACTAAATGA GCAGAGATGG 661 AGTCGACTGA ATAAACAAAG GCGGAGCCAA TTGGATGAGT AGAGGCAGAG CCAGCTAGAT 721 AAGTAGACCC AATGTTGAGT GGAGATAGGT CATGCCAGTT AACAAGACTT GAGACCTAAC 781 CATGGTTTTG TCGATGGAAC TCATTGGTAC GAAGGTTGAG CCTCTGCTCA TGGTCGAATG 841 GAAGGATGAC AGATGGCTTG ATGACACTCC AAGTTCAGGT GTCACGAGCT GGTGCATTAG 901 GTGGGTCTAG CTAGAGAATC TAGCATGCAC GATTGTAGCG ACGTAGACTT CTGCTGTTGG 961 ATTTACTCTT GGATAGCTTA GTTGTCCACT CGGATGGCAG TGTTCTCATC CTCCAAGGCC 1021 TTGATCTTGT TTGTTGTGTC TTTCACTTGT GTGGTGAGGG CAGTGATCTA GGTTGCAATG 1081 TCGTATGAAG GAGTTTCTAT GATTCCGATC ATGACACAGG TCATCCATGC GAAGCAAGTC 1141 GTTGATAGCT GGATGATTGT TAAGGCCCTA TGGTGGGGCC AAATTGTAAA CGGTGTAAGC 1201 TACTTGGAAT TTTGTAAGGA TGATTTTGTA GGGAGATGCC AC

Figure 1. DNA sequences of the female-specific marker FS1 and FS281. A. Annealing positions of the RAPD primer S1 are boxed. The primers designed for amplification of female-specific fragments are indicated by arrows. B. Annealing positions of the RAPD primer S281 are boxed. The primers designed for amplification of femalespecific fragments are indicated by arrows. 


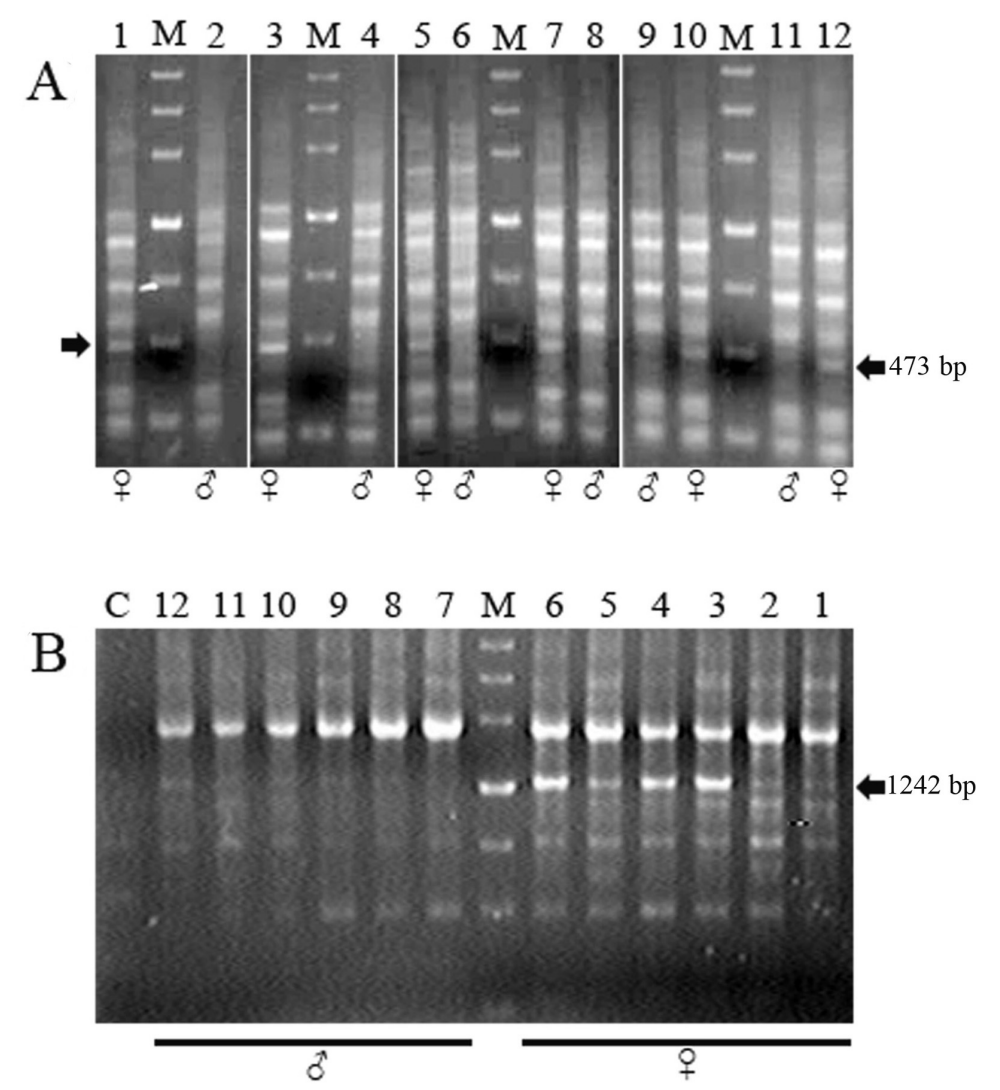

Figure 2. Amplification profiles of RAPD markers by the primers S1 (A) and S281 (B) in Pistacia chinensis. Lanes $M=$ DNA ladder. A. Lanes 1, 3, 5, 7, 10, $12=$ female individuals. Lanes 2, 4, 6, 8, 9, $11=$ male individuals. B. Lanes 1-6 = male individuals. Lanes 7-12=female individuals. Lane $C=$ PCR result in the negative control group without DNA template.

\section{Sex-specific DNA sequences and SCAR marker testing}

DNA sequencing results indicated that the FS1 fragment was 57.4\% AT and 42.6\% GC, while the AT and GC contents in the FS281 fragment were 53.6 and 46.4\%, respectively. Sequence similarity that was searched using Basic Local Alignment Search Tool (BLAST) (www.ncbi.nih.gov) showed no highly homologous sequence for both FS1 and FS281.

The designed SCAR primers were used to test 16 different individuals, including 8 male trees and 8 female trees. The results showed that a single band could be amplified nonspecifically in all female and male specimens using primer S1-1 and primer S1-2 (Figure 3A), and altering the annealing temperature could not change the results, which proved that the SCAR primers that were designed for FS1 were unsuccessful. However, a sex-specific band was amplified using primers S281-1 and S281-2 in all 8 female specimens, which was absent in 8 male specimens when the annealing temperature was $64^{\circ} \mathrm{C}$ (Figure 3B). The reproducibility of results was checked by repeating the reaction twice under the same conditions. 

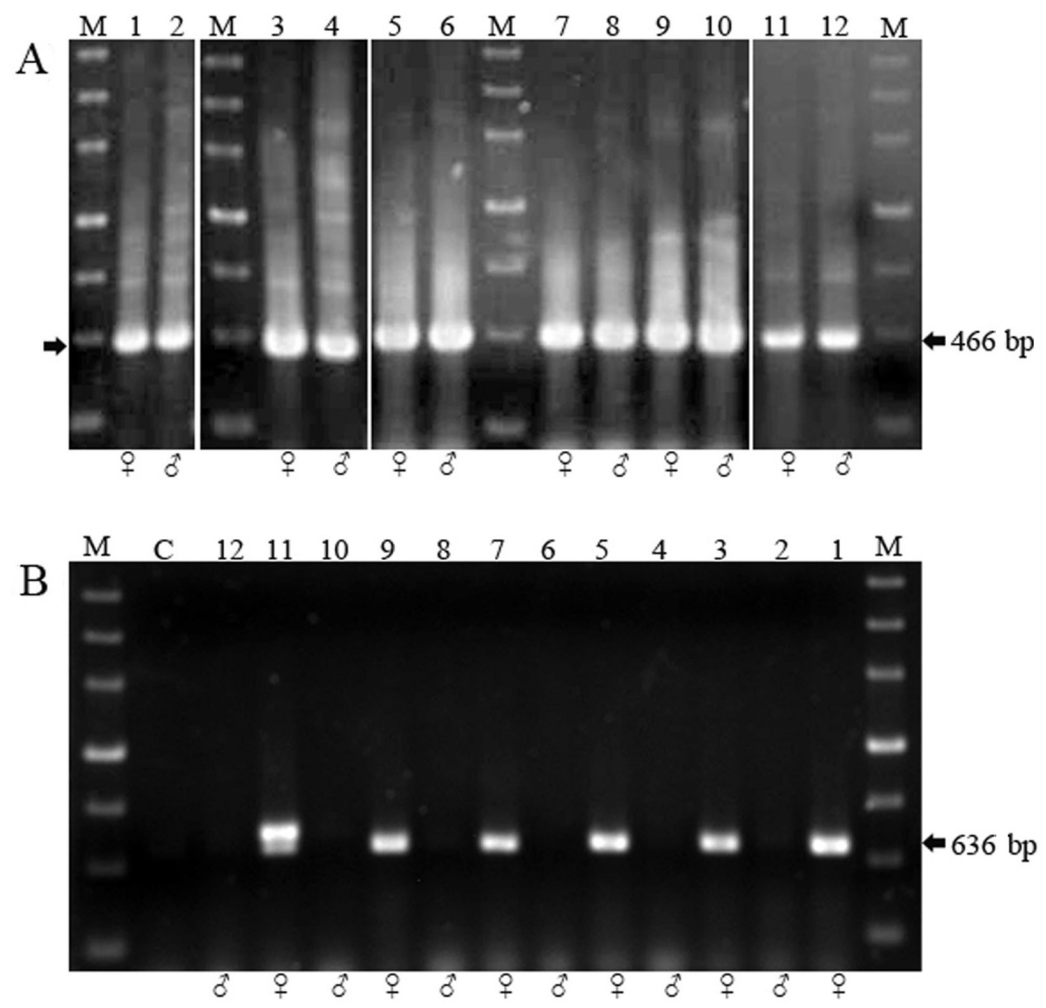

Figure 3. Amplification profiles of SCAR markers by the primers S1-1/S1-2 (A) and S281-1/S281-2 (B) in Pistacia chinensis. Lanes $M=$ DNA ladder. A. Lanes 1, 3, 5, 7, 9, $11=$ female individuals. Lanes 2, 4, 6, 8, 10, $12=$ male individuals. B. Lanes $1,3,5,7,9,11=$ female individuals. Lanes $2,4,6,8,10,12=$ male individuals. Lane $C=$ $\mathrm{PCR}$ result in the negative control group without DNA template.

Over the past decade, the sex-determination of flowering plants by a molecular mechanism was always an attractive research field, and a number of achievements have been made in dioecious plants such as Silene latifolia (Taylor et al., 2001; Hobza et al., 2006; Kejnovsky and Vyskot, 2010; Macas et al., 2011). At present, morphologically distinctive sex chromosomes have been found in 13 plant species, of which the sex-determination mechanism can be divided into 2 groups: the X-to-autosome ratio and the active Y-system. The active Y-system, which is applied by S. latifolia, was similar to a mammalian sex determination mechanism.

Unlike S. latifolia (Caryophyllaceae), which has clearly distinguished X and Y chromosomes (Vyskot and Hobza, 2004; Kejnovsky et al., 2009), the chromosome nuclear type in P. chinensis has not been elucidated. Al-Saghir (2010) reported that the chromosome number of $P$. chinensis was $24(2 n=24)$, but whether a sex chromosome existed was still unknown. In this study, we developed a sex-specific SCAR marker (female-specific) to discriminate the sex of $P$. chinensis, and this might also contribute to the research of the sex determination mechanism of the Pistacia genus. 


\title{
ACKNOWLEDGMENTS
}

\author{
China.
}

Research supported by the Basic Research Project of Qingdao (\#12-1-4-2-(1)-jch),

\section{REFERENCES}

Al-Saghir MG (2010). Perspective on chromosome numbers in the genus Pistacia L. (Anacardiaceae). Int. J. Plant Breed. Genet. 4: 153-157.

Heideman C, Munhoz RE, Pattaro Júnior JR and Fernandez MA (2010). Genetic diversity analysis with RAPD linked to sex identification in the sugar cane borer Diatraea saccharalis. Genet. Mol. Res. 9: 2343-2348.

Hobza R, Lengerova M, Svoboda J, Kubekova H, et al. (2006). An accumulation of tandem DNA repeats on the Y chromosome in Silene latifolia during early stages of sex chromosome evolution. Chromosoma 115: 376-382.

Kejnovsky E and Vyskot B (2010). Silene latifolia: the classical model to study heteromorphic sex chromosomes. Cytogenet. Genome Res. 129: 250-262.

Kejnovsky E, Hobza R, Cermak T, Kubat Z, et al. (2009). The role of repetitive DNA in structure and evolution of sex chromosomes in plants. Heredity 102: 533-541.

Li X, He XY, Li ZL, Wang YD, et al. (2012). Enzymatic production of biodiesel from Pistacia chinensis bge seed oil using immobilized lipase. Fuel 92: 89-93.

Macas J, Kejnovský E, Neumann P, Novák P, et al. (2011). Next generation sequencing-based analysis of repetitive DNA in the model dioceous plant Silene latifolia. Plos One 6: e27335.

Maki M (2009). Development of SCAR markers for sex determination in the dioecious shrub Aucuba japonica (Cornaceae). Genome 52: 231-237.

Qin SJ, Sun YZ, Meng XC and Zhang SX (2010). Production and analysis of biodiesel from non-edible seed oil of Pistacia chinensis. Energ. Explor. Exploit. 28: 37-46.

Renner SS and Ricklefs RE (1995). Dioecy and its correlations in the flowering plants. Am. J. Bot. 82: 596-606.

Taylor DR, Olson MS and McCauley DE (2001). A quantitative genetic analysis of nuclear-cytoplasmic male sterility in structured populations of Silene vulgaris. Genetics 158: 833-841.

Vyskot B and Hobza R (2004). Gender in plants: sex chromosomes are emerging from the fog. Trends Genet. 20: $432-438$.

Yakubov B, Barazani O and Golan-Goldhirsh A (2005). Combination of SCAR primers and Touchdown-PCR for sex identification in Pistacia vera L. Sci. Hortic. 103: 473-478. 